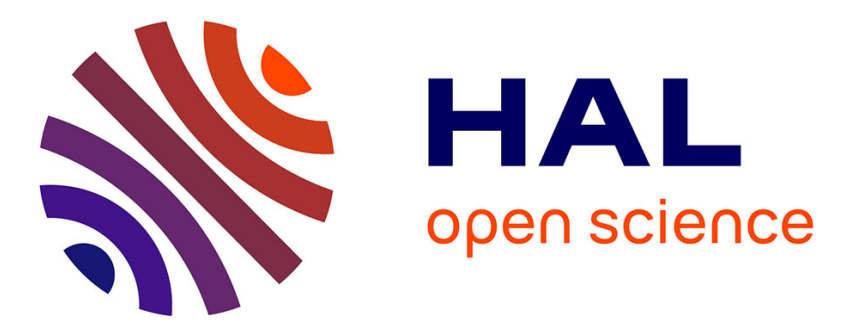

\title{
Figuration, ou la parole éclipsée : le statut discursif de l'accusé dans les procès de cour d'assises
}

Evelyne Saunier

\section{To cite this version:}

Evelyne Saunier. Figuration, ou la parole éclipsée: le statut discursif de l'accusé dans les procès de cour d'assises. Les Acteurs du discours: de l'énonciateur à l'acteur social, Lambert Lucas, A paraître. hal-01739744

\section{HAL Id: hal-01739744 \\ https://hal.parisnanterre.fr/hal-01739744}

Submitted on 21 Mar 2018

HAL is a multi-disciplinary open access archive for the deposit and dissemination of scientific research documents, whether they are published or not. The documents may come from teaching and research institutions in France or abroad, or from public or private research centers.
L'archive ouverte pluridisciplinaire HAL, est destinée au dépôt et à la diffusion de documents scientifiques de niveau recherche, publiés ou non, émanant des établissements d'enseignement et de recherche français ou étrangers, des laboratoires publics ou privés. 


\title{
Figuration, ou la parole éclipsée : le statut discursif de l'accusé dans les procès de cour d'assises
}

\author{
Évelyne Saunier \\ Université Paris Descartes - MoDyCo
}

\section{Introduction}

Le crime est un acte déviant qui se signale par la gravité qui lui est conférée dans une société donnée à une époque donnée. Ainsi, le viol est devenu un crime, et inversement la profanation d'objets religieux ne l'est plus.

«[...] il ne faut pas dire qu'un acte froisse la conscience commune parce qu'il est criminel, mais qu'il est criminel parce qu'il froisse la conscience commune. Nous ne le réprouvons pas parce qu'il est un crime, mais il est un crime parce que nous le réprouvons. » (Durkheim 1970 : 91)

Il y a donc une forme de circularité : est un crime ce qui se juge en cour d'assises, la cour d'assises est l'instance qui juge les crimes (différenciés des délits et infractions).

« [Les crimes] s'attaquent aux fondements-mêmes de l'ordre culturel, aux différences familiales et hiérarchiques sans lesquelles il n'y aurait pas d'ordre social. Dans la sphère de l'action individuelle, ils correspondent donc aux conséquences globales d'une épidémie de peste ou de tout désastre comparable. ils ne se contentent pas de relâcher le lien social, ils le détruisent entièrement. » (Girard 1982 : 27)

Après l'instant de l'acte criminel, et le temps de l'instruction, vient le moment du procès. La forme ritualisée et les spécificités de ce procès public, et parfois fortement médiatisé, se justifient par sa double fonction : pratique (quantification de la peine) et symbolique. Cette dernière consiste en un travail de suturation de la déchirure faite au contrat social, et de réaffirmation de la vitalité de la conscience collective, consolidant ainsi la solidarité entre les membres de la société.

«[...] les actes que [le droit répressif] prohibe et qualifie de crimes sont de deux sortes : ou bien ils manifestent directement une dissemblance trop violente entre l'agent qui les accomplit et le type collectif, ou bien ils offensent la conscience commune. [...] C'est cette force [de cohésion sociale] que le droit pénal protège contre tout affaiblissement, à la fois en exigeant de chacun de nous un minimum de ressemblances sans lesquelles l'individu serait une menace pour l'unité du corps social, et en nous imposant le respect du symbole qui exprime et résume ces ressemblances en même temps qu'il les garantit. » (Durkheim 1970 : 96)

«L'image que l'on se fait du crime et du criminel est la résultante de deux dimensions distinctes : la gravité perçue des conséquences de l'acte et l'intentionnalité attribuée à l'auteur. Et le tout est sous-tendu par un mécanisme encore plus général : la possibilité ou l'impossibilité d'identification. Le "petit délit", on aurait pu le commettre ; le "gros", en aucun cas. En d'autres termes, tout se joue au niveau du droit à la différence que l'on reconnaît à autrui, c'est-à-dire en fin de compte de la marge au-delà de laquelle la différence fait peur. » (Robert $1972: 176)$

Comme l'a montré M. Foucault, depuis le XIXe siècle ce n'est plus la punition (le sacrifice public) qui est le lieu de la catharsis, mais le jugement : la société ne se réunifie plus dans l'émotion partagée de la vengeance et le spectacle de la souffrance infligée, mais dans le spectacle d'une institution qui, jaugeant, évaluant, met en œuvre la raison. Il s'agit toujours de communier dans la reconnaissance publique de la légitimité de la réaction sociale à la déviance. Dans cette mesure, le procès d'assises a quelque proximité avec le théâtre. ${ }^{1}$

«Assister à un spectacle, c'est structurer les logiques d'identité que la représentation donne à voir et reconstruire les logiques esthétiques et symboliques qui, à chaque spectacle, refondent le contrat social grâce à une représentation distanciée de l'identité. » (Lamizet 2002 : 73)

\footnotetext{
${ }^{1}$ Certes le public doit rester coi durant l'audience, mais le rire est toléré, et n'est pas exceptionnel.
} 
Le procès d'assises est donc un des lieux où la société peut se regarder et se réaffirmer dans sa puissance d'agir. C'est aussi ce qu'on pourrait appeler un «moment social total », au sens où s'y manifestent nombre d'institutions et champs sociaux : institutions judiciaire et policière, bien sûr, mais aussi médecine, psychiatrie, sciences humaines, aide sociale... Une grande variété de discours s'y confrontent - pêle-mêle : lecture, rapport d'enquête, plaidoyer, interrogatoire, rapport d'expertise, narration, déclaration performative, commentaire de documents visuels...

Sous tous ces discours émerge parfois l'inentendable, aussitôt éludé et enfoui, ce qui, essentiel, ne peut être clairement énoncé, et qui est montré de longue date par la sociologie : toute société produit sa déviance, le crime est une manifestation du « jeu » allant de pair avec tout système de normes, et les agents du crime sont pris dans des fonctionnements qui les dépassent $^{2}$. À cette approche, qui invaliderait toute tentative de sanction / réparation (buts pratiques du procès d'assises), doit se substituer l'exposition de l'auteur du crime comme sa source singulière.

« On voit poindre en même temps que la nécessité d'une classification parallèle des crimes et des châtiments, la nécessité d'une individualisation des peines, conforme aux caractères singuliers de chaque criminel. [...] La jurisprudence ancienne [...] utilisait pour ajuster le châtiment, deux séries de variables, celles de la "circonstance" et celles de l'"intention". C'est-à-dire des éléments permettant de qualifier l'acte lui-même. [...] Mais ce qui commence à s'esquisser maintenant, c'est une modulation qui se réfère à l'infracteur lui-même, à sa nature, à son mode de vie et de penser, à son passé, à sa "qualité" et non plus à l'intention de sa volonté. » (Foucault $1975: 101-102$ )

Depuis le XXe siècle la criminologie intègre massivement les travaux de la psychologie et s'intéresse à la définition, au repérage et à la rééducation de « la personnalité criminelle ». Ce concept [...] est devenu opératoire [avec la contribution de Jean Pinatel dans un ouvrage de 1963]. [...] Il a [...] opérationnalisé le noyau central de la personnalité criminelle en précisant les quatre traits principaux qui le composent : égocentrisme, labilité, agressivité et indifférence affective. (Le Blanc, $1991: 29)$

C'est le principe d'individualisation de la peine qui justifie le procès, affirmant que l'on ne juge pas des faits désincarnés, mais un homme, une femme. Aussi, tout aussi importante que les actes, sera évaluée la personnalité de l'accusé ${ }^{3}$. Ceci est pleinement assumé, comme le montre ce préambule à l'interrogatoire de l'accusé par le président :

$\mathrm{Pt}$ - alors avant de revenir quelque peu sur les faits on l'a vu vous l'avez vu dans l'arrêt de renvoi ils sont / bien clairement expliqués / je crois qu'il y a (autant que je le sais) il y a pratiquement pas d'ombre sur la façon dont ça s'est passé // mais avant de revenir sur les faits on va essayer / de voir un petit peu comment vous avez vécu jusqu'à présent monsieur /

Dans cette perspective les discours qui s'entrecroisent ont pour fonction la construction d'un personnage de criminel, susceptible d'être saisi dans son être, et que l'on pourra dès lors condamner (ou éventuellement acquitter). Par personnage, nous entendons un individu porteur d'une histoire et d'un "caractère" à partir desquels on peut interpréter ses comportements.

L'observation (avec transcription fine) de divers procès ${ }^{4}$ nous a permis de dégager les éléments linguistiques caractéristiques contribuant à cantonner l'accusé dans un rôle d'objet montré, plutôt que lui autoriser une réelle position de sujet énonciateur.

\footnotetext{
${ }^{2}$ R. K. Merton (1970) a montré les sources sociales et culturelles de la déviance, remettant en question le primat de la motivation psychologique de la criminalité.

${ }^{3}$ Pour ne pas alourdir, « accusé » représente aussi « accusée » et « accusés » (ils peuvent être plusieurs, par exemple dans les procès pour hold-up).

${ }^{4}$ Une vingtaine de procès tenus entre 1989 et 2016, à Besançon (14), Paris (2) et Bobigny (3). Nous avons sélectionné des procès jugeant des crimes de sang et des holdups avec violence, excluant les procès à
} 
Nous questionnerons d'abord le statut de l'oral dans cette interaction ritualisée particulière qu'est le procès : retrouve-t-on les caractéristiques de l'interlocution, de la discussion, de la conversation?

Puis nous étudierons le statut langagier de l'accusé, dont le mode de présence dans le procès est double : comme actant auquel il est fait référence, en tant que protagoniste du crime ; comme acteur, en tant que protagoniste du procès.

Nous conclurons par une réflexion critique sur la problématique du handicap linguistique.

\section{L'oralité des débats}

\subsection{Le rapport à l'écrit}

Le procès d'assises est essentiellement une construction langagière, les éléments matériels (armes, photos...) étant rares. L'oralité des débats est sacralisée : «La plume est serve, la parole est libre » est l'adage fréquemment invoqué par les professionnels de la justice pour souligner le caractère vivant et humain du procès, face à la froideur de ce que pourrait être une décision de professionnels sur documents administratifs. Or les paroles qui s'échangent s'inscrivent par rapport à une considérable somme d'écrits auxquels il est fréquemment fait recours, «le dossier », accumulation de pièces cotées, contenant entre autres les interrogatoires (policiers et judiciaires) de l'accusé.

Ce dossier, dont la connaissance est partagée par les parties adverses et le président, fournit une garantie d'objectivité. Sa matérialité est très présente (grosses piles de documents sur les pupitres) et il est souvent feuilleté, montré... Les quelques exemples suivants illustrent le poids qui lui est conféré :

$\mathrm{AG}^{5}$ - c'est en toutes lettres dans les rapports [des experts] ;

APC - PN est celui contre lequel il [']accusé] [voudrait/pourrait] se battre / parce que / c'est dans

l'dossier / il [PN] mesure 1,65 mètre il pèse 46 kilos ;

$\mathrm{Pt}$ - enfin vous l'avez dit que vous êtes allé chercher cette plaque [d'immatriculation, fausse] / c'est là /

dans l'dossier ! [frappant le dit-dossier du plat de la main] ;

AG - toute l'accusation /j'ai le dossier / consiste en un sinistre tableau de l'accusé ;

A - je suis très fatigué malade en prison [...] mon problème m'a rendu fou

$\mathrm{Pt}-$ il n'en est pas fait mention dans le rapport

$\mathrm{Pt}$ - lors de la dernière campagne électorale / vous avez milité pour le Front National sans faire partie de ce mouvement

A - je (remarque, dirai tout de même que) là-dessus euh / que la politique n'a rien à voir avec euh mon histoire // euh : je... à cause que le / parti du Front National est un parti bien connu alors / je ne veux pas entrer $X X X$

$\mathrm{Pt}$ - rassurez-vous c'est pas du tout un procès politique que je ferai // y a quand même: / c'est une des: des références $X$...X reprises dans le dossier

La forme du procès d'assises et la composition populaire du jury sont étroitement liées, les jurés étant doublement "ignorant(s)" : culturellement (ils sont supposés ne pas avoir de compétence juridique) et contextuellement (ils ne savent rien de l'affaire en question).

retentissement politique, ou les crimes sexuels sur mineurs dont le huis clos peut être décidé à tout moment. Nos données consistent en des prises de notes sténographiques ou/et à plusieurs mains et recoupées (l'enregistrement étant interdit).

${ }^{5}$ Nous emploierons les abréviations suivantes : $\mathrm{A}=$ accusé $; \mathrm{Pt}=$ président du jury $; \mathrm{AD}=$ avocat de la défense ; $\mathrm{APC}=$ avocat de la partie civile $; \mathrm{AG}=$ avocat général ou procureur $; \mathrm{T}=$ témoin $; \mathrm{PN}=$ prénom + nom d'une personne citée $(\mathrm{P}=$ prénom seul, $\mathrm{N}=$ nom de famille seul). $\mathrm{XXX}=$ passages inaudibles ou incompréhensibles ; $[\ldots]=$ passage non transcrit ; (qu'il , qui l') = hésitation entre deux formes ; les mots entre parenthèse ne sont pas garantis dans leur exactitude. Nous mettons en caractères gras les éléments illustrant notre propos. 
Comme ils n'ont pas accès au dossier, c'est pour eux qu'il faut la re-présenter, et cela se fera en partie sous forme d'extraits (lus ou cités de mémoire) qui seront mentionnés soit comme "parlant d'eux-mêmes", soit comme supports d'exégèse. C'est dans cette possibilité d'interprétation qu'il faut situer la "liberté" revendiquée de la parole par rapport à "la plume" plutôt que dans le rapport à une quelconque instance coercitive.

Ainsi, la scène du procès est-elle l'écume d'une accumulation de discours déjà en circulation depuis parfois des années ${ }^{6}$, avec une part d'écrit oralisé qui contribue à rendre difficiles l'écoute et la compréhension.

\subsection{Les ratés de la communication}

Il y a dans les procès de cour d'assises de l'inaudible et du malentendu.

Nous avons été frappée par les problèmes récurrents de sonorisation et/ou d'élocution : dans la quasi-totalité des procès auxquels nous avons assisté, la question s'est posée. Il faut des protestations énergiques du Président ou de l'un des avocats pour qu'il soit remédié au problème qui nuit à la compréhension de certains protagonistes. C'est fréquemment le cas pour les accusés ${ }^{7}$, cela n'est pas rare pour les témoins, et cela arrive aussi pour les professionnels (experts, enquêteurs de personnalité, policiers...).

$\mathrm{Pt}$ - monsieur $N$ ? vous ne changez pas d'attitude vous reconnaissez intégralement les faits qui vous sont reprochés pour lesquels vous êtes jugé? oui ?

A - [inaudible]

$\mathrm{Pt}$ - il faut essayer de parler un petit peu fort monsieur $N$ [pour] bien vous exprimer / parce qu'il faut que les gens / il faut que les gens / vous connaissent / et comprennent pourquoi vous avez fait ça / d'accord?

$\mathrm{Pt}$ - parlez bien fort et près du micro parce que / comme je le dis toujours l'acoustique n'est pas bonne dans la salle

Expert - [inaudible]

$\mathrm{Pt}$ - on vous entend très mal moi je vous entends à peine et les autres

$\mathrm{AD}$ - alors votre témoignage XX vous êtes moins formelle aujourd'hui sur le fait qu'il y ait deux séries de tirs [...] combien de séries de tirs avez-vous entendues?

$\mathrm{T}$ - je peux pas dire

$\mathrm{AD}$ - mais alors bon vous ne connaissez pas toute la procédure mais /// comment se fait-il que [...] ?

$\mathrm{T}-$ [inaudible]

$\mathrm{Pt}$ - parlez bien dans le micro s'il-vous-plaît

Quant au malentendu, il est pour partie lié à la distance socio-culturelle entre les

protagonistes, spécialement l'ensemble président - avocats et l'ensemble accusé - victime témoins (ces derniers étant fréquemment du même milieu). Nous l'illustrerons par deux exemples, dont le premier, extrait du procès Dominici, est célèbre ${ }^{8}$ :

$\mathrm{Pt}$ - êtes-vous allé au pont?

A - allée ? il y a pas d'allée, je le sais, j’y suis été

$\mathrm{Pt}$-pourquoi il y a eu cette distorsion entre le CES et le lycée ? [l'accusé a été bon élève au CES et mauvais au lycée]

A - mais c'est tout à fait logique monsieur le Président après le CES il y a le lycée

[La question ne sera pas reposée.]

De façon plus subtile, la conception de tel ou tel aspect de la réalité peut diverger, et il est très rare qu'un débat puisse être mené. L'extrait ci-dessous, avec un accusé atypique qui tentait de maintenir une position argumentative, illustre ce genre de malentendu :

\footnotetext{
${ }^{6}$ Par exemple, dans un procès de 1999, on lit un rapport d'examen psychiatrique de l'accusé qui date de 1989.

${ }^{7}$ Paradoxalement, c'est lorsqu'un interprète traduit les propos de l'accusé que ceux-ci sont le plus audibles.

${ }^{8}$ Cité par Barthes, repris des notes publiées par Giono.
} 
$\mathrm{Pt}$ - vous vouliez essayer X...X faire une carrière dans la musique

A - euh d'ailleurs depuis euh mon enfance j'ai toujours euh $X \ldots . .$.

$\mathrm{Pt}$ - alors c'était de la musique euh... XX dans la procédure c'était euh hard rock / rock mais hard rock

A - oui c'est exact

$\mathrm{Pt}$ - de la musique euh j'allais dire violente / bruyante

A - c'est-à-dire que les gens la qualifient comme ça mais c'est une musique: dans un sens aussi elle peut

être très calme hein // vous avez des très belles ballades en hard rock // vous avez des très beaux

morceaux de rock XXXX // par exemple si vous écoutez ma chanson préférée le pénitencier c'est vraiment une belle chanson

[rires]

[Plus tard :]

$\mathrm{Pt}$ - je reviens / sur cette musique un peu à caractère euh / violent les:: experts psychiatres pensent que c'était un / un peu un 'fin un exutoire à votre / à votre violence interne // ça vous permettait de / de vous dépenser de:: de libérer ce fond d'agressivité qu'il y avait (en vous) // qu'est-ce que vous en pensez de ça?

A - c'est-à-dire c'est ce que pen-

$\mathrm{Pt}-X X$

A - c'est-à-dire c'est ce que pensent les experts // mais étant donné que je suis_une personne euh / assez calme /je: d'ailleurs (ça a été, c'était) dur dans le: dans les: enquêtes rogatoires / je suis calme: bon / $\mathrm{Pt}$ - oui mais enfin (je vous signale, vous êtes calme)...

A - si / si j'aime c'te musique c'est parce que / si j'écoute Mozart c'est encore plus violent que ce que: que le hard rock vous voyez bien

[rires]

$\mathrm{Pt}$ - je vous laisse juge de:: de votre appréciation

[Plus tard, première phrase du réquisitoire :]

AG - cet homme nous dit que le divin Mozart avait composé une musique plus agressive que celle du groupe ACDC!

\subsection{Un oral peu spontané}

L'absence de véritable interlocution se manifeste sous deux aspects : le peu de cas qui, en définitive, est fait des difficultés d'intercompréhension, et la rareté des marques caractéristiques d'une pratique langagière dialogale. Nous voulons parler des phénomènes typiques de l'oral spontané ${ }^{9}$ tels que :

- certains marqueurs discursifs, ex : vous savez, vous voyez, hein, quoi ;

- des enchaînements parataxiques, ex : elle pouvait pas, c'était fermé ;

- les séquences co-construites dans un entremêlement des locuteurs ;

- la fluidité des échanges dont les interventions se chevauchent, s'enchâssent, leur fonction pouvant être composite (réactive ou initiative et évaluative, par ex.).

Parmi les types d'interaction verbale, le débat est certes plus formel que la conversation courante. La confrontation et le caractère argumentatif des prises de paroles génèrent néanmoins nombre d'échanges présentant les caractéristiques ci-dessus.

Or, de façon générale, la structure du procès consiste en un agrégat de discours juxtaposés. Soulignons que selon sa personnalité, le président peut user plus ou moins de ces formes caractéristiques. Si les avocats les emploient, c'est de façon rhétorique, en les amplifiant à dessein. Mais pour ce qui est des accusés, la crainte de mal s'exprimer ou de dire ce qu'il ne faut pas conduit majoritairement à des phrases courtes, simples, isolées.

C'est visible spécialement durant l'interrogatoire de personnalité, mené par le Président, qui devrait être «le grand moment » de l'accusé, celui-ci s'exprimant souvent de façon laconique :

$\mathrm{Pt}$ : vous savez quelles sont les raisons du placement? / de votre placement / chez: les époux euh N.? A : (oui , euh) // j'ai appris euh / quand j'ai vu le: dossier d'instruction [silence]

\footnotetext{
${ }^{9}$ Voir Rémi-Giraud 1991, Kerbrat-Orecchioni 2010 (spécialement 111-119), Blanche-Benveniste 2010 (spécialement 50-62).
} 
$\mathrm{Pt}$ : et vous ne: / connaissiez pas / vous saviez pas du tout? avant?

A : ben euh // je savais deux trois choses mais y a des trucs ///

$\mathrm{Pt}$ : et quelles étaient ces deux trois choses?

A : ben / je savais que / ma mère se prostituait // (et , mais) que mon père: / devait sûrement avoir des: problèmes (de, avec la) police

\section{Le statut discursif de l'accusé}

Certes, l'individualisation de la peine, le dernier mot à l'accusé (avant le retrait du jury pour le délibéré) témoignent de la volonté de respecter celui-ci comme sujet. Mais cette notion s'entend de trois façons.

- Sujet comme «ce dont il est question », et à cet égard, l'accusé est le sujet majeur (au moins autant que les faits) du procès. L'accusé comme objet de discours ne cesse d'être mis à distance et portraituré par les différents protagonistes.

- Sujet comme un être humain ayant une subjectivité : des affects, une personnalité singulière... le propos du procès est justement de prendre cette dimension en compte.

- Sujet en tant qu'instance se constituant comme « personne », à travers la construction d'une certaine position énonciative dans et par son discours.

Dans cette dernière acception, nos données montrent que cela n'est possible qu'au prix d'une transgression des normes implicites de comportement langagier qui président au mode de présence attendu de l'accusé.

\subsection{L'accusé actant}

Nous examinerons comment, à travers les modes de référenciation à l'accusé, des opérations d'identification, désignation, qualification, étiquetage, catégorisation... en construisent une certaine figure.

\subsubsection{Types de mention}

Dans la façon dont il est fait référence à l'accusé, donc en position thématique, on observe divers syntagmes nominaux. Certains sont banals : Monsieur N, Prénom Nom, Nom de famille seul, Prénom seul (essentiellement dans les plaidoiries de la défense), et massivement il/elle/ils.

On emploiera également l'accusé :

AG - comment l'accusé alors explique / que le lendemain lorsque les enquêteurs / lui présentent / le couteau $X . . . X$ /il le reconnaît formellement ! / comment il explique ça?

Dans ce cas on a affaire à un mode de représentation de l'accusé en tant qu'acteur social qui ressortit à ce que Theo van Leeuven appelle la catégorisation par fonctionnalisation :

"Functionalization occurs when social actors are referred to in terms of an activity, in terms of something they do, for instance, an occupation or role." (Leeuven 2008, 42)

Mais on rencontre également le pronom on et des emplois du pronom tonique lui en position proclitique :

APC - quand on arrive à [Ville] / monsieur N que fait-il ?// [on = les quatre accusés]

APC - là / en même temps qu'on croit frapper quelqu'un / alors on (n')a pas l'idée de la frapper mais on a

I'idée de la XXX à un point particulièrement vulnérable

Expert - lui ne souffre pas consciemment de sa situation

$\mathrm{Pt}$ - lui ne souhaitait pas (revoir son épouse) et son épouse ne souhaitait pas le revoir

L'emploi de on ramène l'accusé à un type humain. L'emploi du pronom tonique proclitique oppose implicitement l'accusé à d'autres individus. 
Les syntagmes nominaux avec comme déterminant un adjectif démonstratif sont tout à fait remarquables :

$\mathrm{AD}$ - le voilà qui arrive [...] ce gosse ... [plus loin :] $P$ / ce gosse [...]

$\mathrm{AD}$ - qu'ont fait ces godelureaux (sinon attirer l'attention sur eux) ?

APC - cet homme-là X...X un tant soit peu de:: pitié [plus loin : ] ce garçon

Brigadier - cet individu qui posait problème avait été expulsé par ses colocataires

$\mathrm{AD}$ - qu'est-ce que représente / pour cet immigré

Lorsqu'on a un nom qualifiant, une proposition implicite présuppose : «A est un $\mathrm{N}_{\text {qual }}$ ». Avec le désignatif -là, on pointe l'individu auquel il est fait référence comme spécifique, particulier.

Cet effet de pointage (ou «monstration ») est particulièrement saillant dans l'emploi récurrent des pronoms démonstratifs celui-cillà, et ceci, sans qu'il s'agisse d'une reprise anaphorique :

$\mathrm{AG}$ - la personnalité de ces gens-là m'intéresse peu parce que [nous sommes dans un cas de] grand banditisme// (...) ceux-là n'y sont pas conduits

$\mathrm{AD}$ - celui-là il a seulement besoin d'une seule chose c'est d'être soigné / dans un établissement spécialisé $\mathrm{APC}$ - et alors devant (la victime) que fait celui-ci?

$\mathrm{AD}$ - écoutez le poème de celui-ci [lecture du poème] [plus loin :] et c'était la vie de celui-ci qui était en jeu

$\mathrm{APC}$ - quelle est la part de l'intention homicide de celui-ci?

$\mathrm{APC}$ - qu'est-ce que les faits nous disent? eh bien que çui-là il est venu [plus loin] pourquoi celui-là il a pu utiliser sa puce électronique?[plus loin] [l'avocat de la défense tentera] bien sûr de vous dire finalement celui-là est victime

On observe également des structures présentatives qui contribuent à « exposer » l'accusé comme objet de contemplation :

$\mathrm{AD}: N / c^{\prime}$ est ce brave bonhomme

$\mathrm{AD}$ - alors voilà le bonhomme à qui vous avez affaire

AG - comment s'étonner / que nanti de cette personnalité contrastée / [...] P.N. soit devenu un meurtrier // c'est l'homme que vous avez à juger

Toutes ces formes remarquables sont aussi sinon plus abondantes que les occurrences de vous qui posent l'accusé comme interlocuteur coénonciateur.

\subsubsection{Modes de prédication}

Nous observerons dans ce qui suit la façon dont l'accusé fait l'objet de différents modes de représentation à travers différents types d'opérations prédicatives.

L'accusé peut être décrit à travers ses comportements, en tant qu'ils témoignent de quel individu il est. On a alors une structure $S N$ qui :

$\mathrm{AD}$ : PN c'est quelqu'un qui / a rencontré/je veux pas dire son destin parce que c'est un bien grand mot mais en tout cas a rencontré une personne // qui est très importante dans sa vie /[...] c'est j'allais dire sa rencontre avec l'amour / avec la femme comme il ne l'a jamais connue $\mathrm{AG}$ - nous avons affaire à des gens intelligents [...] des individus qui sont le produit d'une sorte de parasitisme social // X...X ces garçons qui avaient $X$ un certain nombre de talents $X$...X qui certes n'ont peut-être pas vécu dans l'opulence // mais quand même / de là à basculer dans des phénomènes de grande délinquance il y a une marge

$\mathrm{AD}$ - c'est pas l'homme qui va participer à une ratonnade

$\mathrm{AD}$ - [à un témoin] était-il quelqu'un qui disait des mensonges?

AG - c'est que PN est un homme qui a deux visages / en tout cas / l'image qu'il donne [...] ne correspond pas uniquement à ce (qu'il est) au fond de son être

$\mathrm{AD}$ - c'est pas quelqu'un qui essaie de mentir c'est quelqu'un qui essaie de trouver les mots

On peut également dire comment il est à travers une qualification, avec être + adjectif:

Expert - il est naïf / adolescent // il semble agir sans préparation 
$\mathrm{AD}$ - il n'est pas menteur / il est psychopathe

$\mathrm{APC}$ - c'est un garçon qui est franc d'un bout à l'autre

$\mathrm{APC}$ - finalement il est autocentré monsieur $N$ / et les autres monsieur $N$ ?

Et enfin on dira ce qu'il est à travers une assignation à la classe de ceux qui ont telle qualité : être un+ nom dérivé d'adjectif :

$\mathrm{APC}$ - je dis que vous mentez / vous êtes un menteur

AG - c'est un oisif

$\mathrm{AD}$ - je plaide pour un immature / pour quelqu'un qui est passif / pour un enfant qui a un quotient intellectuel limite

AD - ben oui / parce que P c'est un insignifiant / c'est un petit

$\mathrm{AD}$ - vous avez à juger un malade / et je ne pense pas que quinze ans de prison puissent soigner un malade

$\mathrm{AD}$ - monsieur c'est un réactif / il sait pas réfléchir

Dans ce cas on a une catégorisation par identification, selon la terminologie de Van Leeuven : "Identification occurs when social actors are defined, not in terms of what they do, but in terms of what they, more or less permanently, or unavoidably, are. I have distinguished three types : classification, relational identification, and physical identification. ${ }^{10 "}$ (Leeuven $2008: 42$ )

\subsection{L'accusé acteur}

En tant que locuteur, le mode de présence de l'accusé est fragmentaire et relativement passif : il n'est jamais, en principe, à l'initiative des interactions et ne fait que répondre à des questions ou à des sollicitations.

Les types d'actes de parole et les marqueurs discursifs qui lui sont caractéristiques contribuent à lui conférer un rôle essentiellement illustratif.

\subsubsection{Types de genres discursifs et d'actes de langage}

Il se trouve que l'accusé est cantonné dans certains types d'actes de langage, de genres premiers ou de styles, sans qu'aucun lui soit officiellement interdit.

Certains, très présents dans le discours des acteurs institutionnels, sont ressentis comme hors de propos : la question (y compris rhétorique), l'argumentation ou la démonstration, l'ironie, la contestation. Ou encore, l'énoncé d'une vérité générale, présent prioritairement dans le discours des avocats, serait particulièrement déplacé dans celui de l'accusé. En voici quelques exemples :

$\mathrm{AD}$ - la justice / c'est je dirais / le juste prix d'une faute

$\mathrm{AD}$ - or, on ne choisit jamais la délinquance

AG - l'homme est le seul artisan de sa destinée / il doit se déterminer pour le bien ou pour le mal

$\mathrm{APC}$ - il a tué ses frères PN / c'est souvent le cas d'ailleurs / un chat tue pas un chien

D'autres au contraire seront attendus ou sollicités : la narration, l'expression des sentiments, l'explication, la réponse de confirmation.

C'est cette dernière qui est le mieux représentée. En effet, la séquence au cours de laquelle on est censé entendre le plus l'accusé est l'interrogatoire de personnalité, mené par le Président.

Or le plus souvent, c'est ce dernier qui raconte, l'accusé se bornant à des propos laconiques et à des « oui » de confirmation. Le passage ci-dessous (dont la longueur est nécessaire à l'illustration de notre propos) est assez représentatif :

$\mathrm{Pt}$ - vous êtes né le [jour mois]: / 19.

\footnotetext{
${ }^{10}$ Notons, en référence à ce dernier type, qu'il arrive que l'aspect physique de l'accusé soit un élément de description : AD -regardez-le il a une grosse tête / il a l'air méchant/regardez-le / faut le tuer il est fou / il a les stigmates d'une vie dure ; APC - on voit plus que son front dégarni
} 


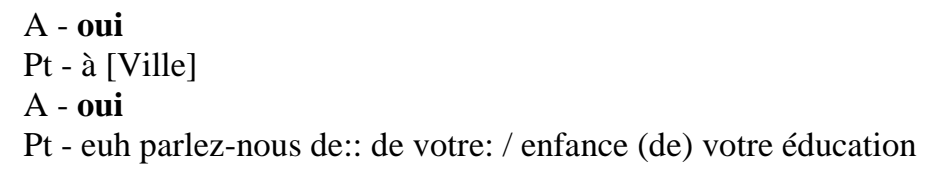

A- ben euh / disons que / quand j'étais chez (les) N. / j'ai pas eu (une,d') enfance malheureuse / (j'ai eu , mais) une bonne éducation

[silence]

$\mathrm{Pt}$ - oui // vous savez quelles sont les raisons du placement ? / de votre placement / chez: les époux euh

N. ?

A - (oui , euh) // j'ai appris euh / quand j'ai vu le: dossier d'instruction

[silence]

$\mathrm{Pt}$ - et vous ne: / connaissiez pas / vous saviez pas du tout ? avant?

A - ben euh // je savais deux trois choses mais y a des trucs

$\mathrm{Pt}$ - et quelles étaient ces deux trois choses?

A - ben / je savais que / ma mère se prostituait // (et , mais) que mon père: / devait sûrement avoir des: problèmes (de, avec la) police

$\mathrm{Pt}$ - ah (oui , bon) / et vous le saviez dep- depuis quand ?

A - euh à peu près depuis l'âge de: / enfin j'en avais entendu parler quand j'avais l'âge de dix ans ou onze ans

$\mathrm{Pt}$ - vous en avez entendu parler par qui ?

A - par (les, des) gens du village /// (puis, et) mon instituteur

$\mathrm{Pt}$ - (alors) / (vous allez, nous allons) en parler tout à l'heure / vous avez été placé (XXX) même / d'un certain nombre de choses qui vous ont. été révélées sur une enfance (dont vous n'avez plus) souvenir soit vous l'avez pas gardé ce souvenir soit vous l'avez: / enfoui // XXXXXX / vous avez découvert vousmême un certain nombre de choses à la lecture du dossier / et notamment je suppose à la lecture de: l'enquête / qui a été faite euh // la synthèse en quelque sorte (depuis votre, de votre ) naissance jusqu'à votre majorité // euh par la DDASS

A - hm / oui

$\mathrm{Pt}$ - hein / on vous a / vous avez vu que:: // comment dirais-je vous aviez été placé // euh / que votre mère avait séjourné euh / en maison maternelle / hein / en 1970 // à ce moment-là elle était séparée / de votre père // (qui était:) / parti de son domicile / sans laisser d'adresse /// à l'époque il aurait_été recherché luimême par la police // euh que:: / votre mère avait. une vie relativement itinérante / instable / hein / semble-t-il

A - oui

Pt - euh donc euh vous: vous trouviez vous-même euh / tout petit à six mois déjà dans une situation / (temporelle, temporaire) / XXXXXXX / hein ? / vous êtes confié à une famille d'accueil ensuite: / euh / euh // votre mère est hospitalisée // elle // habite à droite à gauche // et puis en:: / novembre $71 / \mathrm{XXX}$ l'âge d'un an / euh: au cours d'une crise nerveuse / que / qu'a votre mère // euh: / ben / voilà (ce qu'on nous, ce qui est) dit au cours d'une violente crise nerveuse XXXX danger elle veut tuer son enfant celui-ci / lui est retiré et elle-même est conduite par un pompier au CHR ///à cette époque-là elle semble (attachée à la) vie /mais::/ /son état de santé ne lui permet pas manifestement ///et de sorte que vous allez être placé à l'âge de quinze mois chez les X...X chez lesquels vous resterez jusqu'à l'âge de dix-huit ans A - oui

La narration est souvent sollicitée, mais les accusés que nous avons vus y sont malhabiles. C'est pourtant ce qui pourrait faire pencher le portrait vers une figure avantageuse, comme en témoigne cet extrait relatant un moment du procès Dominici :

«L'accusé est debout. Il est en plein débat avec le président, ses fils et une de ses filles qui sont à la barre. Débat assez monté de ton. Brusquement l'accusé se détourne (...) Il regarde dans le vide (...) Et il parle. Il ne s'adresse à personne. C'est un monologue gratuit. Dès les premiers mots, tout le monde se tait et écoute. Et là, (...) en sept ou huit phrases extrêmement simples et composées avec les mots d'un vocabulaire restreint à l'extrême, l'accusé parle de sa vie bucolique. Le mot est à sa place : c'était aussi beau que du Virgile. (...) Je n'en déduis pas qu'il est innocent : j'en déduis qu'il est sensible. Je ne fais pas son procès : je fais une étude de caractère. » (Giono, 144-145)

Il en va de même pour l'explication et l'expression du ressenti de l'accusé au moment des faits, de l'instruction, de l'incarcération.

De par la stratification des discours, la glose, la reformulation ou l'explicitation sont des genres discursifs omniprésents dans les procès d'assises. Il faut parler sur un dire antérieur 
(datant de plusieurs années comme d'un autre moment du procès) et l'on demande "des développements" ou une interprétation des propos tenus. Cette pratique langagière demande un entraînement, une aisance linguistique qui semble faire défaut, le plus souvent, aux accusés.

$\mathrm{Pt}$-vous avez dit [au cours d'un interrogatoire antérieur] que vous n'appreniez rien dans ces stages... A - ///non c'est-à-dire c'était / des jeunes / au chômage / il fallait qu'ils les placent quelque part ils les mettaient / dans ces stages

$\mathrm{Pt}$ - c'était une relation sérieuse? [avec une femme n'ayant rien à voir avec l'affaire]

A - oui bien sûr/l

$\mathrm{Pt}$ - et alors...

A - //ben je vous dis oui / c'est tout

$\mathrm{Pt}$ - [Vous avez dit ] «je ne m'entends avec personne, je ne sais pas pourquoi»

A - /// ben / avec eux en général / je m'entends pas

Or, les énoncés à commenter ou éclaircir sont aussi ceux qui sont consignés dans le dossier, énoncés que la transcription a pu déformer, comme en témoigne la comparaison de deux extraits de l'ouvrage de Sylvie Péju, qui a pu assister à des interrogatoires dans le bureau d'un juge d'instruction.

Déclarations notées "sur le vif" par l'auteure :

«(...) Le soir, elle a eu un coup de téléphone à onze heures, alors j'ai compris qu'elle avait d'autres relations, j'ai été blessé... Quand je l'ai appelée de Casablanca elle m'a dit : «Viens, viens je t'attends». Je savais pas, moi, elle m'a trompé devant la glace.

(...) Je me rappelle pas comment je me suis jeté sur elle. Elle m'avait dit que c'était fini, j'ai rien dit, elle a parlé, parlé, je n'ai pas réagi et je me suis trouvé plein de rage, ça augmentait ; je ne contrôlais plus mes nerfs...» (Péju 1987, 181)

Texte dicté par le juge d'instruction à la greffière :

«Elle m'a trompé parce que, lorsque je l'ai appelée de Casablanca, elle ne m'a pas dissuadé de revenir. je suis revenu, j'ai couché avec elle et au petit matin, elle m'a parlé, parlé, et plus elle me parlait, plus je me rendais compte qu'elle avait changé et qu'en fait elle était plus la même. Elle m'a signifié que tout était fini entre nous et pendant qu'elle me parlait, je devenais de plus en plus nerveux » (Péju 1987, 184)

La non équivalence entre elle ne m'a pas dissuadé de venir et elle m'a dit : Viens, viens je t'attends» est manifeste. Plus subtilement, la différence entre j'ai rien dit, elle a parlé, parlé et elle m'a parlé, parlé n'est pas négligeable, quand on connaît l'importance des relations victime-accusé dans les débats, le rajout de ce me présentant une parole adressée à l'accusé, que celui-ci ne décrit pas comme telle.

L'illusion de la communication est entretenue par une conception de la langue comme ensemble d'items lexicaux à signification transparente et partagée, aussi la transposition à l'écrit est-elle considérée comme neutre, dès lors que les lexèmes sont identiques.

Certains accusés peuvent avoir nettement conscience du poids de l'écrit et du "glissement" autorisé par la transcription.

A - je sais pas comment la chose a été transcrite mais // j'ai effectivement un souffle au cœur A - elle est bizarre cette déposition // [après lecture de ses déclarations consignées dans le dossier]

Mais il est assez rare qu'ils puissent opérer le redressement qui ramènerait au sens premier, comme dans le cas suivant :

$\mathrm{Pt}$ - vous avez dit «c'est mon blouson»

A - j'ai dit que c'était celui que je portais quand on m'a arrêté // c'est une mauvaise interprétation // effectivement c'est avec ce blouson qu'on m'a arrêté // là je suis formel//

\subsubsection{Marqueurs discursifs privilégiés}


Ceux que nous avons observés fréquemment sont : ben et disons (dans sa valeur d'aveu d'approximation). Ils témoignent d'une forme d'insécurité discursive : puisqu'il faut dire, je dis cela, mais ce n'est pas forcément ce qui serait à dire.

Par contre, d'autres qui abondent dans les discours des protagonistes institutionnels sont très rares : d'ailleurs, par exemple, justement, bon, alors... Ce sont les connecteurs et les baliseurs thématiques dont l'occurrence va de pair avec une relation agentive à la conversation.

\section{Statut interlocutif de l'accusé}

Comme le fait remarquer Antoine Culioli ${ }^{11}$, il est difficile d'attribuer à un interlocuteur des sentiments ou visées qui ne dépendent a priori que de lui. Ainsi, l'énoncé Tu es fatigué, sans autre modalité, est peu attestable. Il le redevient si l'on ajoute Toi (Oh toi (comme je te voislà) tu es fatigué!), ce qui revient, en séparant nettement l'allocutaire - coénonciateur, à introduire explicitement un point de vue d'où l'on se substitue à lui. Si l'on n'opère pas cette manipulation, on déstabilise l'allocutaire en le construisant comme coénonciateur (à travers tu) en même temps que l'on prédique de lui une propriété qui ne saurait l'être que par un sujet énonciateur qui en est en même temps le support.

Or les formules vous êtes + adjectif d'état intérieur sont relativement fréquentes dans la bouche des Présidents. Giono en relève d'ailleurs une occurrence dans ses notes sur le procès Dominici :

«Le Président : Vous êtes très dur.

L'Accusé : Je le suis encore.

Le Président : Indifférent à tout ce qui vous entourait...

L'Accusé : C'était mon travail qui m'intéressait.» ${ }^{12}$

Si la dureté peut être envisagée comme un comportement indépendamment d'une disposition intérieure, ce n'est pas le cas de l'indifférence.

De même, l'expression de la modalité est a priori le fait du sujet modalisateur. Il n'est pas rare que le point de vue de l'accusé soit énoncé par l'un des protagonistes :

$\mathrm{AD}$ - il se sentait proche de son animal // ils se comprenaient / ils ne parlaient pas plus l'un que l'autre $\mathrm{Pt}$ - et alors vous vous vouliez être libéré dans la vie alors (pour,vous) vous libér(er,iez) vous faisiez de la musique / vous vous adonniez à la musique

Greffier [lecture de l'acte d'accusation] - il entre par conséquent dans la vie adulte sans formation / et sans diplôme / cherchant vainement à prouver / qu'il est capable de se débrouiller tout seul

Les formules d'adresse peuvent également contribuer à situer l'accusé hors du champ des instances subjectives reconnues comme telles. Lorsque l'accusé a un interprète, certains présidents formulent les questions à la troisième personne. Cela donne des dialogues comme : $\mathrm{Pt}$ - dernière question euh ses projets d'avenir quels sont-ils? comment voit-il l'avenir ?

A (par l'interprète) - si $\boldsymbol{j}$ 'ai encore le temps pour des années à vivre [...] je vais essayer de rebâtir ma vie $\mathrm{Pt}$ - mais elle est où sa vie?

A (par l'interprète) - rebâtir ma vie en France

Mais il arrive que l'on s'adresse directement à l'accusé à la troisième personne : APC - Pourquoi monsieur $\boldsymbol{N}$ a frappé ?

A - j'ai pris le couteau je lui ai mis un coup ou deux // après je sais pas ce qu'il s'est passé

APC - [citation de l'accusé :] j'ai fumé les cigarettes // combien de temps a-t-il passé ?

\footnotetext{
${ }^{11}$ «Les prédicats subjectifs empêchent qu'un sujet se substitue à un autre sujet. Ainsi Tu as faim est impossible, alors que Est-ce que tu as faim? ou Toi, tu as faim! (au sens de Tu m'as l'air d'avoir faim) sont, évidemment, licites. » (Culioli 1999, 95)

${ }^{12}$ (Giono 1955, 17) Soulignons que l'auteur prend soin de préciser : Je note exactement ses réponses.
} 

A - [silence]
$\mathrm{Pt}$ - [sollicite, relance]
A - je sais pas
Pt - qu'est-ce qu'il en pense?
A - j'ai pas vraiment agressé monsieur $N$ [...] je lui ai juste montré le révolver

Or s'adresser à l'allocutaire avec la troisième personne revient à lui conférer un statut de «non-personne », dans l'acception d'E. Benveniste ${ }^{13}$, c'est-à-dire à l'exclure du champ des sujets énonciateurs susceptibles de (re)prendre en charge des opérations prédicatives et énonciatives au sein des énoncés.

Autrement dit si l'accusé est un sujet, c'est en tant que ses caractéristiques de sujet humain (agent potentiel, support d'émotions, de valeurs...) font l'objet de discours, et non en tant qu'il peut se constituer lui-même comme instance énonciative dans un véritable rapport d'interlocution.

\section{Conclusion}

Au terme de cette recherche, nous nous inscrivons en faux contre la thèse du « handicap linguistique » (défendue par Giono, Barthes... et reprise par Bessette), qui pose qu'un manque de compétence langagière nuit à l'accusé - insistant sur « le peu de mots » dont il ou elle dispose.

«L'accusé qui, pour s'exprimer, même pour défendre sa tête, ne dispose que d'un vocabulaire de trente à quarante mots au maximum, ne comprend pas ces phrases claires et ne peut pas en prononcer de semblables mais il a entendu le nom : Clovis. Il s'intéresse à la discussion.» (Giono 1955, 62) «Ce clown [Maillet, un témoin] a cependant un vocabulaire de cent à cent cinquante mots. L'accusé n'a qu'un vocabulaire de trente à trente cinq mots, pas plus. (J'ai fait le compte d'après toutes les phrases qu'il a prononcées au cours des audiences.) Le président, l'avocat général, le procureur, etc., ont, pour s'exprimer, des milliers de mots.» (Giono 1955, 67-68)

«Tout accusé disposant d'un vocabulaire de deux mille mots serait sorti à peu près indemne de ce procès. Si, en plus, il avait été doué du don de parole et d'un peu d'art du récit, il serait acquitté. Malgré les aveux.» (p 74) (Giono 1955, 74)

«Le président d'Assises, qui lit Le Figaro, n'éprouve visiblement aucun scrupule à dialoguer avec le vieux chevrier "illettré". N'ont-ils pas en commun une même langue et la plus claire qui soit, le français ? Merveilleuse assurance de l'éducation classique, où les bergers conversent sans gêne avec les juges ! Mais ici encore, derrière la morale prestigieuse (et grotesque) des versions latines et des dissertations françaises, c'est la tête d'un homme qui est en jeu. [...] Nous sommes tous Dominici en puissance, non meurtriers, mais accusés privés de langage, ou pire, affublés, humiliés, condamnés sous celui de nos accusateurs. Voler son langage à un homme au nom même du langage, tous les meurtres légaux commencent par là.» ( Barthes 1957, 54-56).

«Qu'y a-t-il de commun entre le langage (code restreint) du vieux berger qui, pendant toute la durée du procès, n'a utilisé qu'un vocabulaire de 35 mots, et celui (code élaboré) du président ou des avocats qui disposent de milliers de mots. [...] Ainsi l'appareil judiciaire, au service du pouvoir d'une classe, est investi par un langage dominant. En l'occurrence, ce mécanisme par lequel l'idéologie bourgeoise submerge le monde du prolétaire s'exerce à travers le mythe de la transparence et de l'universalité du langage.» (Bessette 1982, 101-2)

Ceci repose sur une représentation lexicaliste de la langue qui sous-tend la perception et l'évaluation des discours sociaux. Plus généralement, l'idée qu'une compétence discursive

\footnotetext{
13 « [...] la différence entre l'énonciation « subjective » et l'énonciation « non subjective » apparaît en pleine lumière, dès qu'on est avisé de la nature de l'opposition entre les « personnes » du verbe. Il faut garder à l'esprit que la « $3^{\mathrm{e}}$ personne » est la forme du paradigme verbal (ou pronominal) qui ne renvoie pas [c'est l'auteur qui souligne] à une personne, parce qu'elle se réfère à un objet placé hors de l'allocution. » (Benveniste 1966, 265)
} 
serait favorable à l'accusé et ferait pencher la balance du côté d'un allégement de la peine est assez répandue :

«The more knowledgeable and competent a public speaker is, the better the speaker's chances to influence truth allocation. This sociocultural capital (in the form of reputation, interactional skills, and access to discourses genres) depends upon sociocultural factors [...] which are asymmetrically distributed.» (Jacquemet 1996, 190)

Or, c'est contradictoire avec tout ce que nous avons pu observer. Ainsi, Dominici, condamné à la peine de mort sans preuve formelle, sur la foi de ses seuls aveux rétractés, se révèle-t-il un interlocuteur extrêmement offensif et retors. Citons quelques passages transcrits par Giono qui en témoignent :

L'Accusé - Alors, monsieur le Président, vous me croyez coupable? [Réponse non notée du Président sur son objectivité de principe.] L'Accusé - Mais vous me le faites bien comprendre.

L'Accusé - Je vous écoute, monsieur le Président, dites toujours. (p. 20)

Le Président - Si ces accusations étaient fausses, ce serait ignoble. L'Accusé - Vous pouvez le dire!

L'Accusé - Vous m'en demandez trop, monsieur le Président.

(...)

L'Accusé - Vous pouvez dire tout ce que vous voulez, monsieur le Président, moi je ne sais pas, j'étais couché. (p. 21)

Le Président - Vous étiez très sévère. L'Accusé - Oui. Alors, si un père n'est pas sévère, qui le sera ? (p. 24)

Le Président - Vous ne laissez rien paraître de vos émotions. L'Accusé - Pour quoi faire ? Le Président Les psychiatres disent que vous êtes normal. L'Accusé - Diable! Je ne suis pas fou, non!

Le Président, un peu énervé. Il le sera rarement. - Laissez-moi parler. L'Accusé. - Laissez-moi parler moimême. Je ne veux pas passer pour un autre. Monsieur le Président, si on vous prenait comme on m'a pris, nous verrions ce que vous feriez. J'écoute ce que vous "disez", vous devez écouter ce que je dis. (p. 2526)

L'Accusé - Eh! oui, monsieur le Président. Il faut bien qu'il y ait quelque chose comme ça. Pourquoi un fils accuserait son père? Je le comprends comme ça. (p. 46)

De même, l'un des procès de notre corpus était celui d'un accusé atypique qui parvenait à rectifier, défendre ses positions, exprimer un avis sur le monde. Il a été condamné au maximum requis par l'avocat général. Ou encore, Knobelspiess qui était un accusé combatif et disert a toujours été condamné lourdement, sauf dans le procès dont la fin nous est décrite en ces termes :

«[...] le 18 janvier 1986, Roger Knobelspiess, accusé d'un hold-up qu'il a toujours nié, s'était écrié, la voix entrecoupée de sanglots, juste avant que les jurés ne se retirent pour délibérer : « Je suis innocent, je voudrais pouvoir revoir ma mère et lui apporter des croissants tous les matins. » Un juré s'était mis à pleurer. Quelques heures plus tard, sans qu'on puisse naturellement préjuger de l'impact de ce dernier épisode du procès, Roger Knobelspiess était acquitté. » (Vernier 1989, 70)

Notons encore que tout autant que l'atrocité de ses crimes, ce qui a choqué et a nui à Pierre Rivière est d'avoir rédigé un mémoire de quarante pages :

«[... le mémoire] a pris place [...] parmi les autres éléments du dossier. Chacun semble avoir considéré qu'au lieu d'éclairer ou d'expliquer le crime, il en faisait partie. [...] Il semble que le jury ait jugé plus monstrueux qu'insensé le fait d'avoir joué ce jeu familier [le récit de crime, genre discursif en vogue au XIXe siècle] à la fois dans le texte et dans le geste, d'en être le double auteur et d'y figurer comme le double sujet. » (Foucault 1973, 265 et 274)

Les verdicts et l'attitude des Présidents montrent clairement que « le bon accusé » est celui qui illustre au mieux son portrait en se cantonnant à l'incarnation d'une figure saisissable. Son registre est celui de l'émotion, non de la raison. La bonne stratégie est celle d'une division du travail entre l'avocat de la défense, qui prend en charge le raisonnement et la contradiction, et 
l'accusé qui se présente sous le meilleur jour possible - tel l'objet en vitrine disposé sous le meilleur éclairage. L'important est qu'il abandonne son essence de «personne », et ce faisant s'abandonne, s'offre comme objet.

Un accusé polémiste ou moraliste subvertit l'édifice et dès lors, sa parole joue contre lui. Un mode de présence discursif actif, mettant en avant une position de locuteur-énonciateur raisonnable, lève le voile sur la fragilité de la frontière entre tout citoyen, participant de ce nous qui va juger, et ceux qui s'en trouvent exclus.

L'accusé ne peut donc (ni ne doit) adopter une véritable stratégie d'acteur. Le cadre du procès ne s'y prête pas car il n'autorise pas le déploiement d'une véritable parole, qui opposerait à son identité réifiée de criminel une identité de sujet en relation avec d'autres sujets.

\section{Bibliographie}

BARTHES Roland, 1957, Mythologies, Paris, Seuil.

BENVENISTE Émile, 1966, Problèmes de linguistique générale, Paris : Gallimard (TEL). BESSETTE Jean-Michel, 1982, Sociologie du Crime, Paris, P.U.F. (Le Sociologue).

BLANCHE-BENVENISTE Claire, 2010, Approches de la langue parlée en français, Paris : Ophrys.

CULIOLI Antoine, 1999, Pour une linguistique de l'énonciation. Domaine notionnel, Tome 3, Paris, Ophrys.

DURKHEIM Émile, 1970, «Définitions du crime et fonction du châtiment», in SZABO Denis, Déviance et Criminalité, Paris, Armand Colin.

FOUCAULT Michel (éd.), 1973, Moi, Pierre Rivière, ayant égorgé ma mère, ma sœur et mon frère... Un cas de parricide au XIXe siècle, Paris, Gallimard/Julliard (Archives).

FOUCAULT Michel, 1975, Surveiller et punir. Naissance de la prison, Paris, Gallimard (NRF).

GIONO Jean, 1955, Notes sur l'Affaire Dominici, Paris, Gallimard (NRF).

GIRARD René, 1982, Le Bouc émissaire, Paris, Grasset.

JACQUEMET Marco, 1996, «Power Alliances in Court. Turning Turncoats into Witnesses», Folia Linguistica, 30 (3-4), 189-215.

KERBRAT-ORECCHIONI Catherine, [1990] 2010, Les Interactions verbales, Tome 1, Paris : Armand Colin.

LAMIZET Bernard, 2002, Politique et identité, Paris, Presses Universitaires de Lyon.

LE BLANC Marc, 1991, " La personnalité délinquante », in CARIO Robert \& FAVARD Anne-Marie, La personnalité criminelle, Actes des journées Pinatel, Paris, Érès, 27-46. MERTON Robert K., 1970, « Structure sociale, anomie et déviance », in SZABO Denis, Déviance et criminalité, Paris, Armand Colin, 132-164.

PÉJU Sylvie, 1987, Palais de Justice, Paris, Seuil (L'épreuve de faits).

RAYMONDIS Louis-Marie, LE GUERN Michel, 1976, Le Langage de la Justice Pénale, Paris, Éditions du CNRS.

REMI-GIRAUD Sylvianne, [1987] 1991, «Délimitation et hiérarchisation des échanges dans le dialogue », in COSNIER Jacques et KERBRAT-ORECCHIONI Catherine, Décrire la conversation, Presses Universitaires de Lyon, 17-72.

ROBERT Philippe, 1972, La sociologie entre une criminologie du passage à l'acte et une criminologie de la réaction sociale, Paris, S.E.P.C.

VAN LEEUVEN Theo, 2008, Discourse and Practice : New Tools for Critical Analysis, Oxford, Oxford University Press. 
VERNIER Dominique, PEYROT Maurice, 1989, La Cour d'Assises, Paris, PUF (Que saisje ?). 